\title{
C-SYMMETRIC OPERATORS AND REFLEXIVITY
}

\author{
KAMILA KLIŚ-GARliCKA AND MAREK PTAK
}

Abstract. We study subspaces of all $C$-symmetric operators. Description of the preanihilator of all $C$-symmetric operators is given. It is shown that the subspace of all $C$-symmetric operators is transitive and 2-hyperreflexive.

Mathematics subject classification (2010): Primary 47A15; Secondary 47L99.

Keywords and phrases: $C$-symmetric operators, preanihilator, reflexivity, hyperreflexivity.

\section{REFERENCES}

[1] W. T. ARVESON, Interpolation problems in nest algebras, J. Funct. Anal. 20 (1975), 208-233.

[2] J. B. CONWAY, A course in operator theory, AMS, Graduate studies in mathematics; v. 21.

[3] S. R. Garcia, M. Putinar, Complex symmetric operators and applications, Trans. Amer. Math. Soc. 358, 3 (2005), 1285-1315.

[4] K. KLIŚ, M. PTAK, k-hyperreflexive subspaces, Houston J. Math. 32, 1 (2006), 299-313.

[5] J. KRAUS, D. LARSON, Some applications of a technique for constructing reflexive operator algebras, J. Operator Theory 13 (1985), 227-236.

[6] J. Kraus, D. R. Larson, Reflexivity and distance formulae, Proc. London Math. Soc. 53 (1986), 340-356.

[7] W. E. LongstafF, On the operation Alg Lat in finite dimensions, Lin. Alg. Appl. 27 (1979), 27-29.

[8] J. R. Ringrose, Compact non-self-adjoint operators, Van Nostrand-Reinhold, New York, 1971. 